\title{
Development of Thermal Sensors and Drilling Systems for Application on Lunar Lander Missions
}

\author{
Norbert I. Kömle · Erika S. Hütter • Günter Kargl • Hehua Ju • \\ Yang Gao $\cdot$ Jerzy Grygorczuk
}

Received: 12 December 2007/ Accepted: 18 September 2008/Published online: 7 October 2008

(C) The Author(s) 2008. This article is published with open access at Springerlink.com

\begin{abstract}
The upcoming lunar lander missions, for example Chang'e 2 from CNSA and several mission proposals and studies currently under consideration at NASA (e.g. Neal et al., ROSES 2006 Proposal to NASA, 2006), ESA (e.g. Hufenbach, European Workshop on Lunar Landers, ESTEC, Noordwijk, The Netherlands, 2005; Foing, EPSC Abstracts, vol 2, EPSC2007-A-00422, European Planetary Science Congress, Potsdam, Germany, 2007) and JAXA, Japan (Matsumoto et al., Acta Astronautica, 59:68-76, 2006) offer new possibilities to measure the thermal properties of the lunar regolith and to determine the global lunar heat flow more accurately than it is hitherto known. Both properties are of high importance for the understanding of the lunar structure and the evolution of the Moon-Earth system. In this paper we present some work on new thermal sensors to be used for in situ investigations of the lunar soil in combination with novel drilling techniques applicable for the lunar regolith. Such systems may preferably be mounted on mobile stations like the lunar rover currently built for the Chinese Chang'e 2 mission. A general description of a presently tested prototype of the lunar rover is given and mounting possibilities for a drilling system and thermal sensors are shown. Then we discuss some options for thermal sensors and drills and how they could be combined into one compact instrument. Subsequently a tube-like sensor suitable for measuring the thermal conductivity of the material surrounding a borehole
\end{abstract}

Presented at the 9th International Conference on Exploration and Utilization of the Moon, ICEUM9/ILC2007, Sorrento, Italy, 22-26 October 2007.

N. I. Kömle $(\bowtie) \cdot$ E. S. Hütter · G. Kargl

Space Research Institute, Austrian Academy of Sciences, Schmiedlstrasse 6, 8042 Graz, Austria

e-mail: norbert.koemle@oeaw.ac.at

H. Ju

School of Electronic Information and Control Engineering, Beijing University of Technology (BJUT), Beijing, China

Y. Gao

Surrey Space Centre, University of Surrey, Guildford, UK

J. Grygorczuk

Space Research Centre (SRC), Polish Academy of Sciences, Warsaw, Poland 
is described in more detail. Finally the performance of such a tube-shaped sensor when applied in a lunar borehole is investigated by thermal modelling and compared with the behaviour of a more conventional needle-shaped sensor.

Keywords Regolith · Thermal probes · Drilling · Lunar rovers

\section{Introduction}

The upper layers of a planetary body have a large influence on the thermal history of the body as a whole, since they form the boundary between the planetary interior and free space. On Earth, the so-called geothermal temperature gradient has been studied extensively and measured down to depths of several thousand metres at many places (NOAA Borehole data and Climate Reconstruction Data Base 2005). Such data form the basis for detailed modelling of the temperature distribution in the Earth's interior and its variation during geologic history. They also play a big role for understanding the global and local energy balance of the layered Earth (Haenel et al. 1988). The key material parameter controlling the geothermal temperature gradient is the thermal diffusivity $\kappa$, which is connected with the bulk material density $\varrho$, the heat capacity $c$ and the thermal conductivity $\lambda$ via the relation

$$
\kappa=\frac{\lambda}{\varrho c}
$$

$\lambda$ usually exhibits big variations with depth, because it is highly dependent on the texture and the porosity of the material (Wechsler and Glaser 1965).

On rocky bodies without an atmosphere, like the Moon or asteroids, the variations of $\lambda$ and $\varrho$ from the surface towards the interior can be much larger than on Earth, since these bodies are covered by a porous "rubble pile" layer usually termed regolith, which is thought to act like a thermally insulating sheet between the interior of the body and outer space. Therefore the knowledge of the thermal conductivity $\lambda$ and its variation with depth is very important for understanding the thermal budget of these layers and for modelling the thermal history of the whole body (Langevin and Arnold 1977). The importance of heat flow measurements, particularly for the Moon, was also emphasized in a recent report of the US National Research Council (2006).

Heat flux and thermal conductivity measurements are best done in boreholes of a few meters depth. Up to now they have only been performed successfully at two places on the Moon, in the frame of the Apollo 15 and 17 missions (Langseth et al. 1972, 1976). These pioneering in situ measurements showed that the uppermost layers of the lunar regolith have an extremely low thermal conductivity, which is probably caused by the loose packing and irregular shape of the lunar soil particles (Pilbeam and Vaisnys 1973). Also for the first time numbers for the interior heat flux of the Moon were obtained. However, the results of these Apollo measurements, being local measurements at two selected places, are by far not enough to create a global picture of the lunar heat flow and of the variation of thermal properties as a function of surface location and depth. The new lunar lander missions planned for the coming years offer an opportunity to perform a lot more in situ thermal measurements either by using a network of many miniature geophysical packages (as currently investigated in a NASA study, see Neal (2006)) or by using mobile stations as the Chang'e 2 lunar rover, which is part of the Chinese lunar research program (Ju et al. 2006). We discuss designs for thermal measurements in boreholes on the Moon based on 
the heritage of recent developments for the Rosetta/Philae mission, where the experiment MUPUS will do thermal measurements on a comet nucleus surface (Spohn et al. 2007).

There are three components to be considered.: (i) a thermal conductivity sensor suitable to measure the very low conductivity to be expected with some depth resolution, (ii) a deployment system for the sensor, and (iii) a suitable drilling device. We propose an instrument combining a drill and a thermal sensor that may be operated from a rover or a stationary lunar lander. Novel drilling techniques, as described e.g. by Gao et al. (2007) or Kömle et al. (2008b) may allow to produce boreholes of up to $2 \mathrm{~m}$ depth in the regolith with a reasonable time and power budget, while the proposed deployment system for the thermal sensor may at the same time help to stabilize the borehole. In order to allow accommodation within the limited space available on a rover, a flexible system is proposed, which can be stored on a compact spool when not in use, but behaves like a stiff rod in deployed position. A system similar to that developed for the deployment of the MUPUS thermal probe (Spohn et al. 2007) offers a suitable solution to fulfill these requirements.

In order to measure the thermal conductivity of the material surrounding the borehole in different depths, a transient method like the "heated needle probe" appears most useful (Kömle et al. 2007, 2008a). However, special attention must be spent on the geometry of the sensor to be used. First it has to be assured that the sensor has a good contact to the walls of the borehole, since a vacuum gap can significantly affect the accuracy of the measurement. Secondly, since a several metres deep borehole cannot be made extremely thin over its full length, the thermal sensor should be designed as a hollow cylinder heated along its mantle surface rather than as a thin needle, unless it can be attached to the front side of the drillbit.

The paper is organized as follows. First we consider the scientific requirements and the technical constraints for thermal measurements in boreholes on the lunar surface in a general way and identify realistic options. Then some currently planned missions are described that may provide new bore platforms on the Moon in the near future. Subsequently we review shortly novel drilling techniques that may have the potential for creating reasonably deep and slender boreholes in the lunar regolith, while still complying with a restricted mass and power budget. The main part of the paper is devoted to thermal sensors suitable to be combined with a driller/sampler system. We describe a recently developed prototype including a deployment device and present finite element model calculations illustrating the performance of the two considered thermal sensor designs, a tube-shaped and a needle-shaped sensor.

\section{Thermal Measurements in Boreholes}

\subsection{Scientific Requirements}

For thermal measurements on a planetary body two items are of primary interest:

(1) The thermal conductivity and diffusivity of the material in different depth layers, and

(2) the steady state heat flux from the interior, undisturbed from surface temperature variations caused by solar irradiation.

At which depth can we expect such undisturbed conditions on the Moon? Clearly, this depends on the surface temperature variations, which in turn depend on the periodicity of the radiation. In the case of the Moon this period $P$ is approximately 28 days. Then, if the material properties appearing in Eq. 1 are known, the so-called thermal skindepth can be calculated as: 


$$
D=\sqrt{\frac{\kappa P}{\pi}}=\sqrt{\frac{\lambda P}{\pi \varrho c}}
$$

Table 1 summarizes the main parameters of the regolith in a depth of $1 \mathrm{~m}$. The listed values are mainly retrieved from the papers collected in the Lunar Sourcebook (Heiken et al. 1991) which reflect the state of knowledge after completion of the Apollo missions. With the material parameters given in Table 1 one obeys a typical skin depth of $D \approx$ $0.1 \mathrm{~m}$. As the plots in Fig. 1 show, there is not much deviation from this value if one uses the $\min / \max$ values for $\varrho$ and $\lambda$ listed in Table 1 , a few percent at most.

\subsection{Technical Constraints}

The technical constraints faced when developing a drill for the lunar regolith concern two items:

- the depth of the borehole, and

- the diameter of the borehole.

Both are mainly constrained by the limited weight, volume and power budget usually available. For determining the global heat flux it is necessary to measure the temperature gradient over some distance in a depth below several thermal skin depths. While measuring over a distance of tens of meters would be desirable, the above analysis shows that with accurate temperature sensors $1 \mathrm{~m}$ measuring length in a depth below $1 \mathrm{~m}$ might be enough to assess the interior heat flux. Thus choosing $2 \mathrm{~m}$ drilling depth as a design goal seems to be a reasonable compromise.

Concerning the drill hole diameter, a slender hole would be most desirable for thermal measurements, because it causes the smallest disturbance to the mechanical texture of the surrounding regolith and to the natural temperature field in the regolith. This would

Table 1 Representative values for lunar regolith parameters in a depth of $1 \mathrm{~m}$

\begin{tabular}{|c|c|c|c|c|}
\hline Property & Min value & Max value & Average value & Reference \\
\hline Bulk density & $1300 \mathrm{kgm}^{-3}$ & $1300 \mathrm{kgm}^{-3}$ & $\begin{array}{l}1825 \mathrm{kgm}^{-3} \text { Value in } \\
1 \mathrm{~m} \text { depth calculated } \\
\text { from formula given } \\
\text { in the reference }\end{array}$ & $\begin{array}{l}\text { Carrier III (1991): LSB } \\
\text { p. 493; (Lunar } \\
\text { Source Book) }\end{array}$ \\
\hline Porosity & 0.52 & 0.46 & 0.5 & $\begin{array}{l}\text { Carrier III et al. (1991) } \\
\text { LSB p. 492; }\end{array}$ \\
\hline $\begin{array}{l}\text { Thermal } \\
\text { conductivity }\end{array}$ & $0.015 \mathrm{~J} \mathrm{~kg}^{-1} \mathrm{~K}^{-1}$ & $0.03 \mathrm{~J} \mathrm{~kg}^{-1} \mathrm{~K}^{-1}$ & $0.023 \mathrm{Wm}^{-1} \mathrm{~K}^{-1}$ & $\begin{array}{l}\text { Vaniman (1991): } \\
\text { LSB pp. 36-38; }\end{array}$ \\
\hline $\begin{array}{l}\text { Heat capacity } \\
\quad \text { (basalt) }\end{array}$ & & & $840 \mathrm{~J} \mathrm{~kg}^{-1} \mathrm{~K}^{-1}$ & Allen et al. (1994) \\
\hline $\begin{array}{l}\text { Mean particle } \\
\text { size }\end{array}$ & $40 \mu \mathrm{m}$ & $800 \mu \mathrm{m}$ & $100 \mu \mathrm{m}$ & $\begin{array}{l}\text { McKay et al.(1991): } \\
\text { LSB pp. 304-306; }\end{array}$ \\
\hline $\begin{array}{l}\text { Compaction } \\
\text { strength }\end{array}$ & & & $220 \mathrm{kPa}$ & $\begin{array}{l}\text { Schultz and Siddhartan } \\
\text { (2007) }\end{array}$ \\
\hline Cohesion & $2.4 \mathrm{kPa}$ & $3.8 \mathrm{kPa}$ & $3.0 \mathrm{kPa}$ & $\begin{array}{l}\text { Carrier III et al. (1991) } \\
\text { LSB p. 510; }\end{array}$ \\
\hline $\begin{array}{l}\text { Internal friction } \\
\text { angle }\end{array}$ & $41^{\circ}$ & $55^{\circ}$ & $49^{\circ}$ & $\begin{array}{l}\text { Carrier III et al. (1991) } \\
\text { LSB p. 510; }\end{array}$ \\
\hline
\end{tabular}




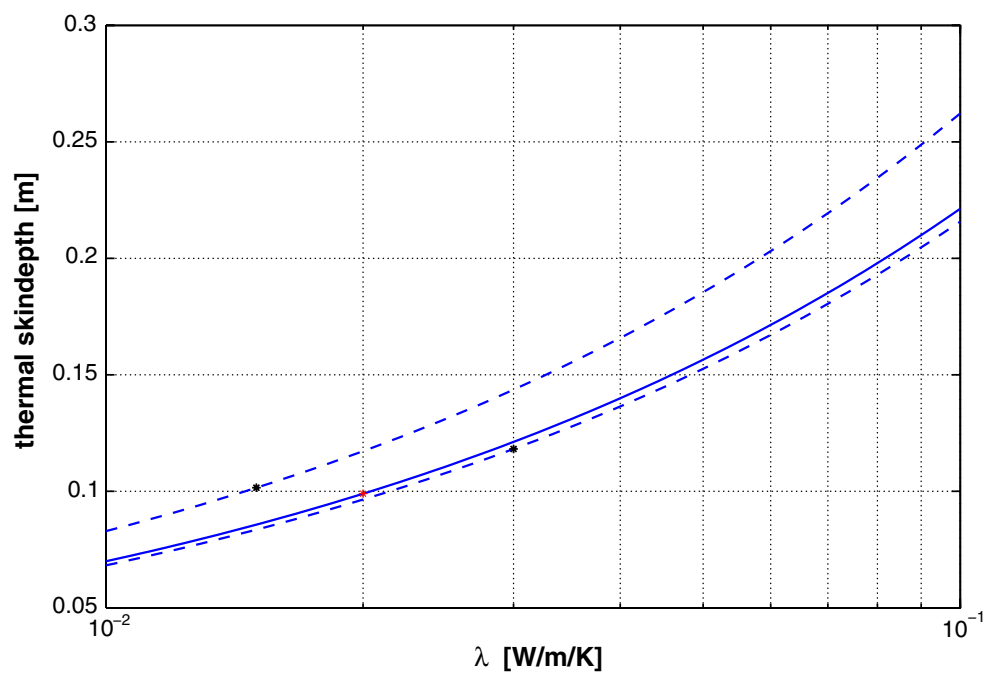

Fig. 1 Variation of the lunar regolith skindepth with the material properties. The full curve assumes the average density as given in Table 1, the dashed lines correspond to minimum and maximum values of $\varrho$. The stars indicate minimum, maximum, and average $\lambda$-values given in Table 1

probably be no problem for holes in the depth range of 10-20 cm, but for drilling holes of $2 \mathrm{~m}$ depth most devices may get stalled due to too big wall friction and the tendency of the soil to close the hole above the drillbit. This would not be compatible with a recoverable system. On the other hand, boreholes with diameters in the several $\mathrm{cm}$ size range might become to massive and demand too much power to be used on a lunar rover mission. Thus a reasonable compromise could lie in the size range of $1.5 \mathrm{~cm}$ up to maximal $2 \mathrm{~cm}$ diameter. As the lunar soil has some internal cohesiveness, holes of this size may remain open even after withdrawal of the borestem. This conclusion is supported by the drilling experience reported by the astronauts of the Apollo missions (Heiken et al. 1991).

\section{New Bore Platforms on the Moon}

\subsection{Chang'e 2 Lunar Rover}

The Chang'e 2 lunar mission of CNSA, which is the follow-up mission of Chang'e 1, is currently under development. In contrast to Chang'e 1 it will be a lander mission including two main parts: a stationary lander and a rover. The lifetime of the mission is planned to be about 6 months. Several prototypes have been built for development purposes at various research institutes in China, two of them at the Beijing University of Technology and the Harbin Institute of Technology (Ju et al. 2006).

Figures 2 and 3 show the latest prototype developed at the Deep Space Robot Research Center of the Beijing University of Technology. The main parameters of this rover are listed in Table 2.

The rover is a 6-wheeled vehicle which will be able to navigate autonomously on the lunar surface. This means it must have the ability to recognize obstacles by itself and to find a save way across the lunar surface without interaction with the ground station. It is 


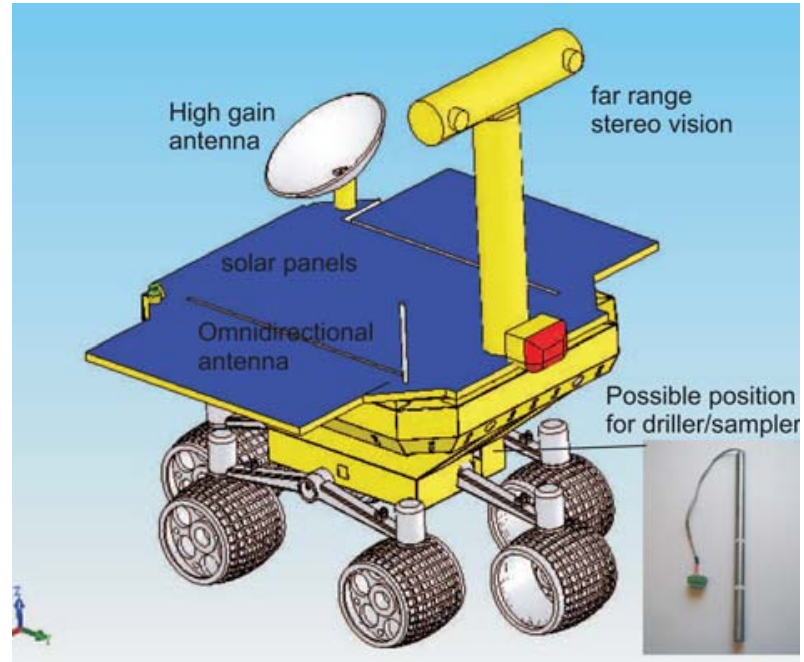

Fig. 2 Prototype BJ2 designed at BJUT for the Chang'e 2 mission. A drilling/sampling system integrated with thermal sensors may become part of the payload of this vehicle

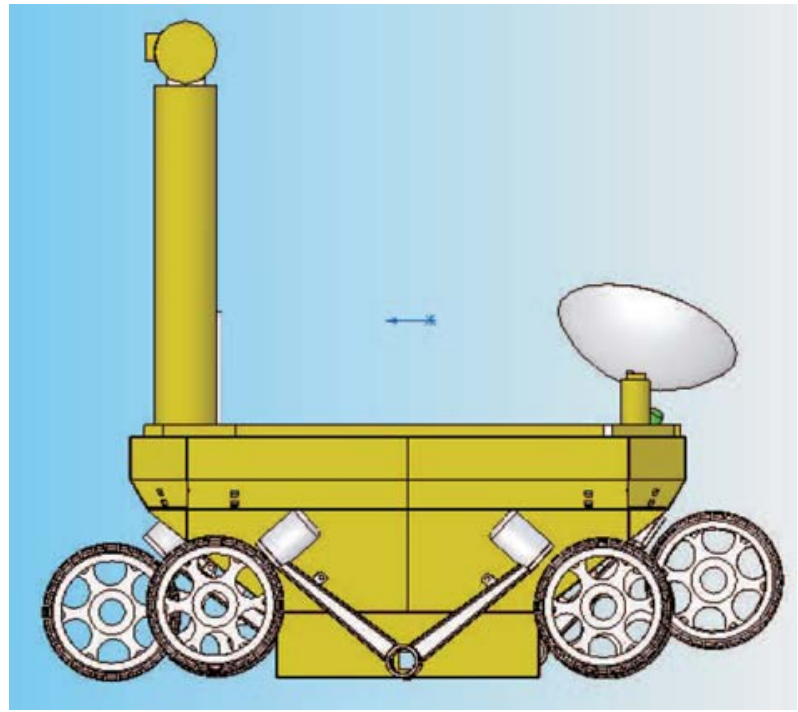

Fig. 3 Side view of the BJ2 rover prototype developed at BJUT for the Chang'e 2 mission, showing more details of the wheels and the rockers connecting the side wheels

equipped with two folding hexagonal rockers on its left and right sides and two folding forks on its rear and front sides. Each wheel is driven by an independent brushless DC motor with a harmonic reducer. Rockers and forks are originally in a folded position to save storage space during transfer to the Moon. After landing they are unfolded by pyrotechnical actuators. The forks at the front and rear sides can also be used to adjust the pitch angle of the rover while it moves across uneven terrain. This design provides 
Table 2 Main design parameters for the Chang'e 2 lunar rover

\begin{tabular}{ll}
\hline Property & Value \\
\hline Total mass (incl. payload) & $90 \mathrm{~kg}$ \\
Total length & $96 \mathrm{~cm}$ \\
Total width & $40 \mathrm{~cm}$ \\
Total height & $94 \mathrm{~cm}$ \\
Wheel diameter & $24 \mathrm{~cm}$ \\
Power budget & $400 \mathrm{~W}$ \\
Full speed & $1.44 \mathrm{~km} / \mathrm{h}$ \\
Maximal slope angle & $30^{\circ}$ \\
Maximal obstacle height & $36 \mathrm{~cm}$ \\
Bottom clearance & $32 \mathrm{~cm}$ \\
\hline
\end{tabular}

consistent normal ground pressure, excellent climbing capability, small stowing volume and high reliability. It should therefore be well suited to cross moderately rough terrain. The rover can traverse ground pits (e.g. small craters) with a diameter of up to $20 \mathrm{~cm}$ and obstacles of up to $36 \mathrm{~cm}$ height. Extensive field tests with the new prototype on a desertlike terrain in China are planned in the near future.

Although the payload of the rover is not yet finally defined, the envisaged strawman payload has already been specified. It is listed in Table 3. Aside of cameras and various spectrometers for characterizing the chemical and mineralogical composition of the regolith it contains also a drilling and sampling system, which can be combined with a thermal probe.

\subsection{Lunar Network Stations}

Further possible platforms for the application of the thermal sensors described in this paper would be the geophysical network stations currently studied in the frame of a NASA funded project, as proposed by Neal (2006). Measurement of the lunar heat flow and the thermal properties of the lunar regolith forms one of the cornerstones of this project. In comparison with a rover mission, thermal measurements aboard such network stations have one important advantage: they are not as time-critical as measurements on a rover, since each station stays on its landing position over the whole lifetime of the mission. With such stations, passive temperature measurements in different depths of the regolith could be done over years on various places and the time interval for active thermal conductivity measurements can be chosen much longer than it is possible on a rover mission.

Table 3 Strawman payload for the Chang'e 2 lunar rover

\author{
Instrument/Device \\ Cameras (stereo) \\ Geochemistry instruments \\ Neutron spectrometer \\ IR-spectrometer $(5-20 \mu \mathrm{m}$ range) \\ Gamma/X-ray spectrometer \\ Dust detector \\ Dosimeter \\ drilling/sampling system
}




\section{Drilling Devices for the Lunar Regolith}

\subsection{Woodwasp Ovipositor Type Drill}

As discussed in the previous section, thermal conductivity and heat flux measurements require drilling to create a deep $(\sim 2 \mathrm{~m})$ and narrow $(\leq 2 \mathrm{~cm}$ in diameter) borehole. This type of deep drilling is very hard to operate in a low gravity environment such as the lunar surface. For example, Apollo astronauts found it difficult to operate a drill manually that went down for $2 \mathrm{~m}$. To perform the same task using an automated drilling device would be even more challenging.

A possible solution could be a bio-inspired drilling device recently studied at Surrey Space Centre (Gao et al., 2007). Biologists (Vincent 1995) investigated digging organisms among insects, such as female wood wasps and female locusts, who use their ovipositor valves to drill into trees or soil to lay eggs. The woodwasp ovipositor utilizes a longitudinal reciprocating motion of a pair of "valves" which slide freely against each other but are held firmly together like the two parts of a "ziplock". When the backwards-facing teeth on one valve snag on the substrate and resist pulling, they generate an equal but opposite force on the other valve to push it into the substrate. A cyclical repetition of this process allows the ovipositor to dig into the substrate without the need for external reaction force. The ovipositor-type drilling mechanism results in a minimum overhead load and low operating power compared to the conventional rotary approach.

Lab-based experiments were set up to verify and demonstrate the feasibility of such a design concept. For these preliminary experiments, a simplified prototype was built, with a drill bit diameter of $18 \mathrm{~mm}$ (Fig. 4). So far, it has successfully drilled into three silicatetype simulates with different hardness measured in terms of compressive strength. Test results are shown in Fig. 5. Although to date only shallow holes with a depth of a few centimeters were drilled by this method, these tests have demonstrated the feasibility of the method in various substrates. The drill string deployment mechanism described in Gao et al. (2007), which supports lengthly drilling, is a conceptual design at the moment and has not yet been tested experimentally. This will be part of the future work on drill development at Surrey Space Centre.

\subsection{Pneumatic Drilling}

Alternative designs for lunar regolith drilling systems, utilizing rotary drilling, are, for example, described in Zacny et al. (2006). A particularly promising development, which
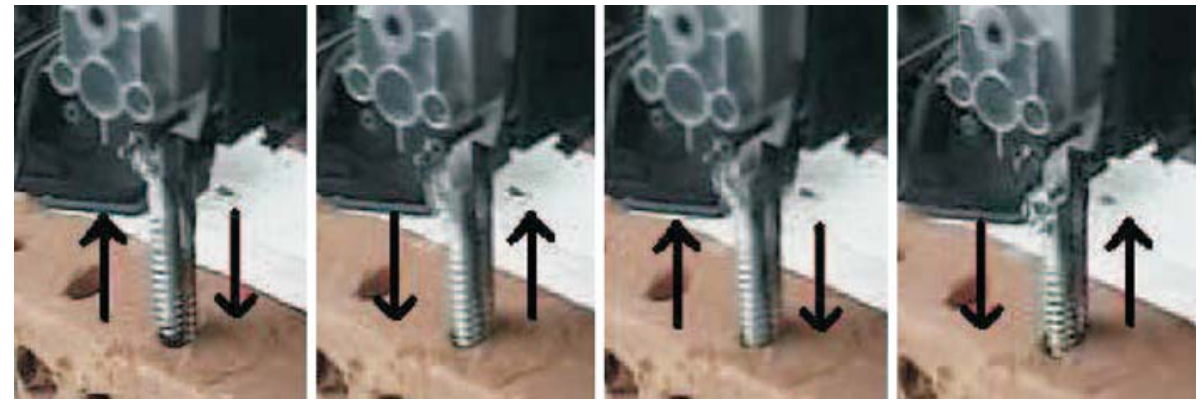

Fig. 4 Laboratory tests with the woodwasp drill prototype 


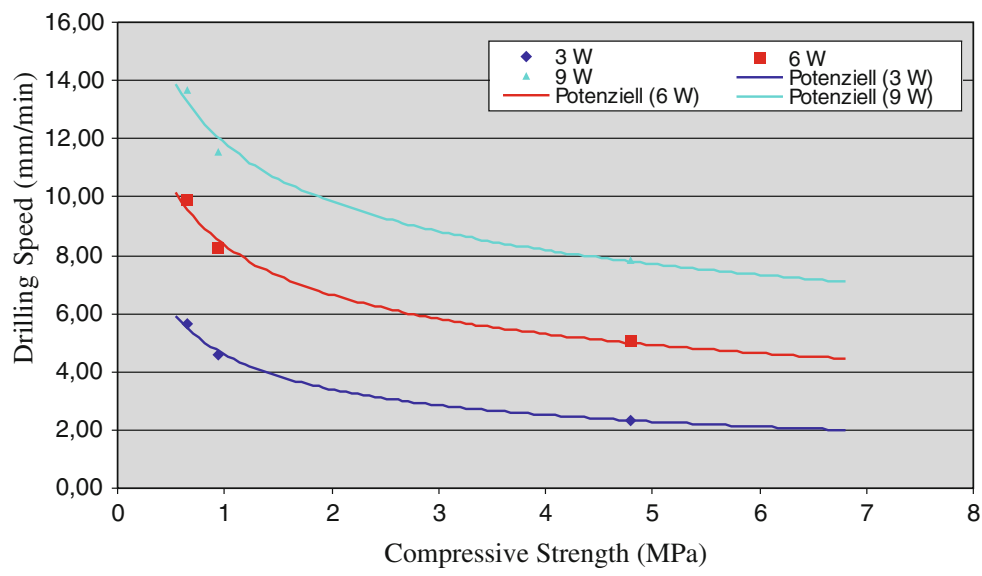

Fig. 5 Test results with the woodwasp drill prototype

might prove useful for the excavation of slender drill holes needed for thermal measurements on the Moon, has recently been proposed by Zacny et al. (2007). It consists of a pneumatic drilling device, where the vertical pushing force is produced by gas pressure from a propellant tank and this gas is at the same time used to remove the drill debris from the borehole through an exhaust pipe. While such a system is also using rotary drilling, there is no need to employ an auger as in conventional drilling systems, because the debris is removed by the gas. This helps to save weight and volume and moreover the gas can be used as a cleaning agent for mechanical parts and joints of the system. Another advantage noted by the authors is that the gas flow could to some extent counteract unwanted heat production inevitably associated with drilling action. This could be particularly important on the Moon, where due to the lack of an atmosphere any use of cooling liquids as usual in terrestrial applications is hardly possible. A feasibility study investigating such a concept for lunar applications has recently been performed by Kömle et al. (2008b).

\section{Deployment Device}

Next we describe a deployment device that was constructed to serve for deploying of any sensor in a boehole with up to $2 \mathrm{~m}$ depth and a diameter of $\geq 16 \mathrm{~mm}$. This deployment device (shown in Fig. 6) works in a similar way as the system mounted on the Rosetta Lander Philae for deployment of the MUPUS penetrator after landing on the comet (Spohn et al. 2007). Note that the woodwasp drill described above uses the same principle for providing a stiff borestem of appropriate length which can nevertheless be stored in a small volume. The deployment device consists of the following main elements:

- A mounting structure for fixing the system mechanically on a rover or lander.

- A spool where a $5 \mathrm{~cm}$ wide stainless steel band of $2 \mathrm{~m}$ length is spooled up. This band is pre-stressed in lateral direction, so that it gets relaxed when the band is wound off the spool. In the relaxed state the band forms a roundish structure of ca. $16 \mathrm{~mm}$ diameter. This structure behaves like a stiff tube and can withstand bending forces that may appear during insertion into the borehole.

- A mechanical interface connecting the deployment device with the thermal sensor. 
Fig. 6 Deployment device for the Hukseflux thermal sensor prototype shown in Fig 7
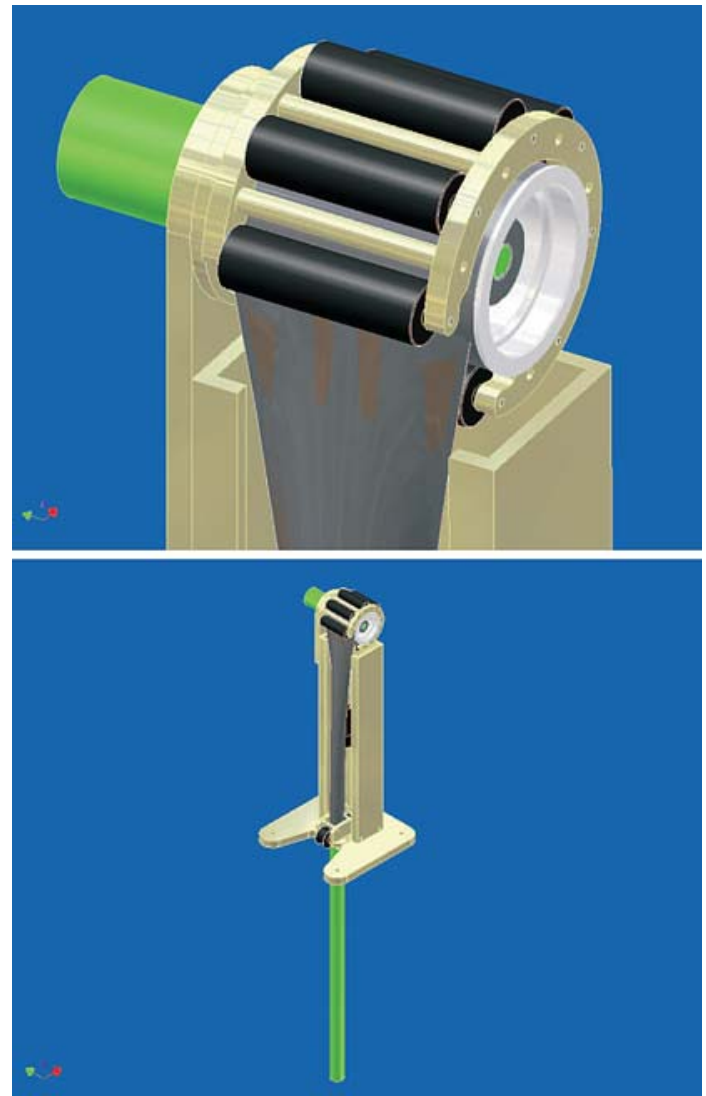

The deployment device is operated by a DC motor with a planetary gearhead, which is used to unwind and upwind the steel tape in order to lower the sensor into the borehole or to retract it.

\section{Thermal Sensor for Lunar Regolith Measurements}

To measure thermal properties of the regolith in different depths below the lunar surface a thermal sensor has to be combined in a smart way with a drill. Depending on the over-all concept of the mission, different scenarios can be chosen:

(1) If the borehole is to be investigated by several different sensors subsequently, a carousel could be mounted in a central position on the rover, which contains along a common work radius both the drill and the various sensors which can be lowered into the borehole after the drilling action (for example a borehole camera, a permittivity probe, and a thermal conductivity sensor. In this case the borehole must be somehow secured against loose debris that may otherwise fall off the walls. From the point of view of a thermal properties sensor this approach has the disadvantage that the size of the borehole needs to be several centimeters and measuring times for determining thermal properties would be high. 
Fig. 7 Prototype of a thermal sensor for measuring thermal conductivity of the lunar regolith. The central hole (with a diameter of $10 \mathrm{~cm}$ in this prototype) may serve as a feed-through for the borestem. Its outer diameter is $16 \mathrm{~mm}$ and its total length is $30 \mathrm{~cm}$

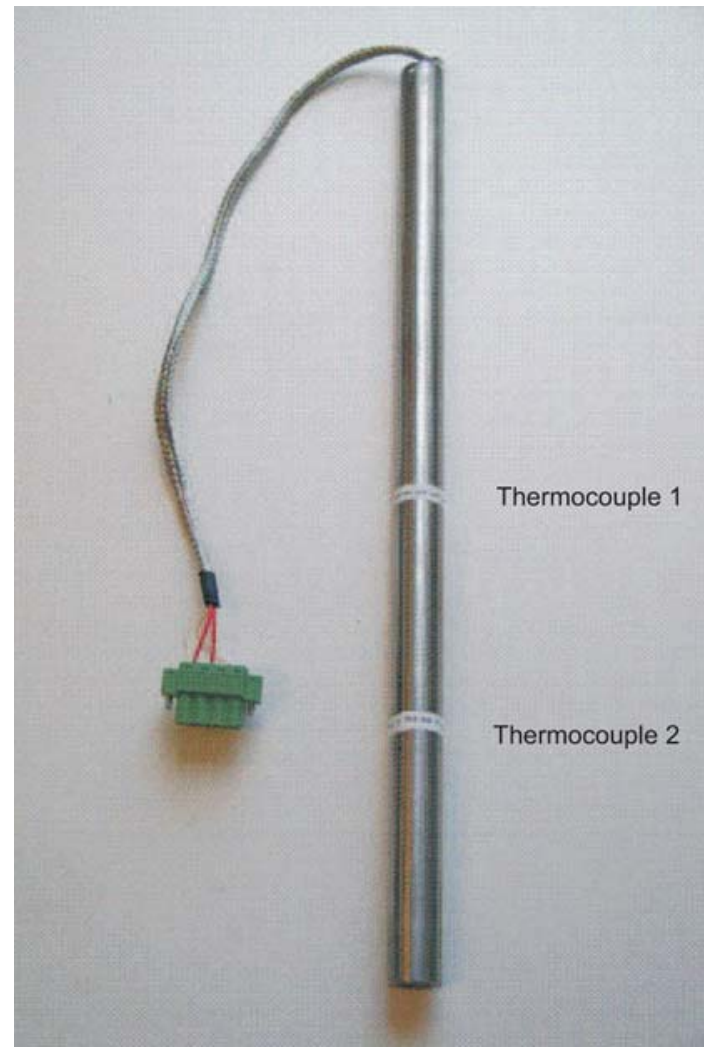

(2) With a moderately thin drill (say around $16 \mathrm{~mm}$ diameter as the heat conductivity sensor prototype shown in Fig. 7) the thermal sensor can be integrated with the drilling device by mounting it above the drill bit. To ensure good contact to the borehole wall during measurements, the sensor can be made of two half lobes, which are pressed apart (against the borehole wall) during the measurements while tightly closing against the borestem during drilling action.

(3) An alternative scenario would be to use a thin needle (similar to the conventional Hukseflux TP02 sensor (http://www.hukseflux.com), but made of stronger material) and to store it in the central part of the drill bit during drilling action, while pushing it forward into the fresh (not yet drilled) soil for a thermal measurement. This would have the advantage that there is always good contact between soil and needle during a measurement without the need of further mechanical action. The disadvantage of this approach is that during insertion the needle may hit large compact particles which cannot be pushed aside easily for further penetration. In this case the needle could not penetrate to the desired depth or may be damaged by bending.

\section{Theory of Thermal Conductance}

In many practical heat conduction problems the thermal contact resistance between adjacent regions consisting of different materials is neglected. This means that a 
continuous temperature change across boundaries is assumed. However, the problem of thermal contact resistance between two media becomes more relevant when heat transfer processes under vacuum conditions are studied. For thermal conductivity measurements on the lunar surface with a sensor integrated in a drilling device as described in the previous section this problem may be particularly severe. Therefore, before describing the results of our model calculations in more detail, we shortly review the formulae used for computing thermal conductance across various boundaries.

This theory is described in detail in the review given by Yovanovich (1998). It is mainly applied for calculating the cooling of electronic devices by attaching solid metal pieces as described e.g. in the paper by Gruijcic et al. (2005). Few work has been published concerning the application of the hot wire method in porous materials of low thermal conductivity. Ebert et al. (1993) give a method to evaluate hot wire measurements in super-isolating materials with conductivities $\lambda \leq 0.01 \mathrm{Wm}^{-1} \mathrm{~K}^{-1}$, which may also be useful for measurements in lunar regolith. Also, with some minor modifications, the formulae given by Yovanovich (1998) should be applicable to the lunar regolith case.

Since the lunar environment is a high vacuum, we neglect all terms which are associated with the heat transfer carried by gases. There remain two contributions, namely the contact conductance $h_{c}$ and the radiation conductance $h_{r}$, which, in the most general form, are given as follows:

$$
\begin{gathered}
h_{c}=\frac{1}{2 \sqrt{2 \pi}} \frac{m}{\sigma} k_{s}\left(1-\sqrt{\frac{p}{H_{c}}}\right)^{-3 / 2} \mathrm{e}^{-\left[\operatorname{erfc}^{-1}\left(\frac{2 \mathrm{p}}{\mathrm{H}_{\mathrm{c}}}\right)\right]^{2}} \\
h_{r}=\left(\frac{1-\varepsilon_{1}}{2 \varepsilon_{1}}+\frac{1-\varepsilon_{2}}{\varepsilon_{2}}+1.104\right) 4 \sigma T^{3}
\end{gathered}
$$

For the derivation of the formulae (3) and (4) refer to Yovanovich (1998) and the references given therein. ${ }^{1}$ The total thermal conductance $h$ is the sum of these two contributions:

$$
h=h_{c}+h_{r}
$$

The contact conductance $h_{c}$ as given in Eq. 3 is a function of the pressure $p$ with which the two surfaces are pressed against each other. In addition it depends on the roughness of the two surfaces $\left(\sigma_{1}\right.$ and $\left.\sigma_{2}\right)$, the root mean square (RMS) inclination of the rough surface elements $\left(m_{1}\right.$ and $\left.m_{2}\right)$, and the microhardness of the softer of the two surfaces, $H_{c}$. Furthermore a harmonic average of the thermal conductivities of the two contacting surfaces is used, $k_{s}=2$ $k_{1} k_{2} /\left(k_{1}+k_{2}\right)$. The RMS roughnesses and inclinations of the two surfaces are defined as

$$
\sigma=\sqrt{\sigma_{1}^{2}+\sigma_{2}^{2}}
$$

and

$$
m=\sqrt{m_{1}^{2}+m_{2}^{2}}
$$

The parameters determining radiative conductance are mainly the infrared emissivity of the two contact surfaces, $\varepsilon_{1}$ and $\varepsilon_{2}$. Since radiation interaction is geometry dependent, it is assumed here that on a microscopic scale the sensor surface is smooth, while the soil surface has an undulating shape consisting of half spheres. The geometrical assumptions underlying the model are illustrated in Fig. 8.

${ }_{1}$ erfc $^{-1}$ is the inverse complimentary error function available as a built-in function for example in the MATLAB software used for our calculations. 


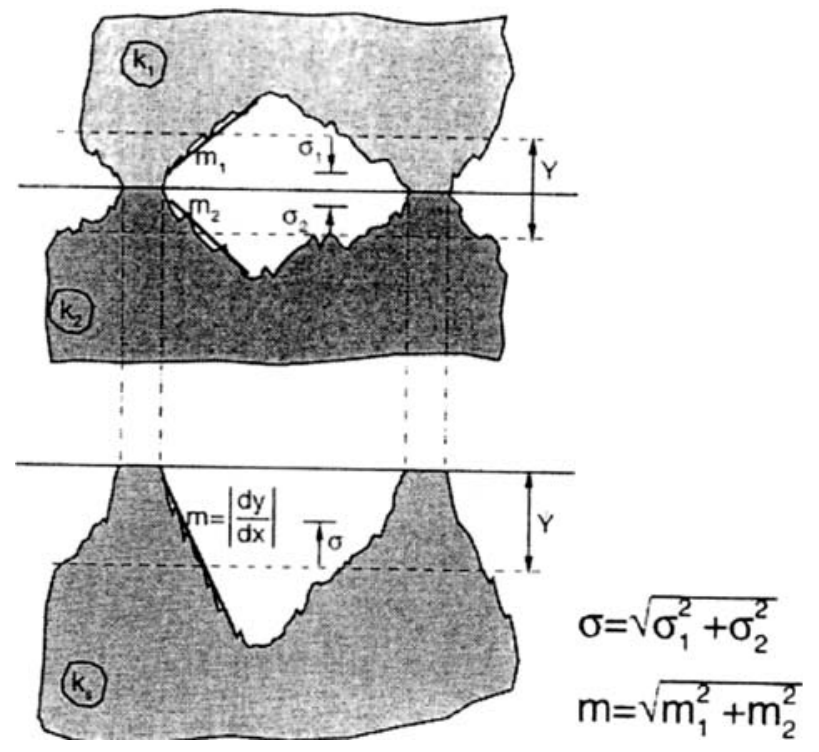

Fig. 8 Illustration of the model assumptions used for calculating the thermal conductance (respectively thermal resistance) between two conforming surfaces (adapted from Yovanovich 1998)

The main difference between the model of Yovanovich (1998) and our application lies in the definition of the microhardness parameter. In the classical theory it determines the resistance of the body against compression of the contacting parts and thus against the increase of the contact surface. In our case we have a contact between a hard, smooth surface (the probe body) and a much weaker, rough surface (the lunar soil). Clearly deformation of the soil in response to pressure will not only be determined by its elastoplastic parameters, but also (and even primarily) by its granular and porous structure. We can safely assume that the strength of individual particles is considerably higher than the strength of the bonds between particles. Therefore, in the absence of a more refined theory, it appears reasonable to use the compaction strength of the lunar soil as the characteristic parameter replacing the microhardness. Instead of surface roughness, the characteristic average particle size of the lunar soil should be the representative length scale to be used. For both parameters typical values are known, which are based on investigations of the Apollo lunar soil samples and from lunar regolith analogue materials. Concerning the compaction strength, Schultz and Siddhartan (2007) have performed soil-mechanical tests with a standardized lunar analog material, leading them to suggest a typical value of $220 \mathrm{kPa}$. The size distribution of the lunar regolith particles is quite well known and described in detail in the Lunar Sourcebook (Heiken et al. 1991). Based on these informations we assume $100 \mu \mathrm{m}$ as the typical particle diameter (corresponding to a roughness scale of $50 \mu \mathrm{m}$ for a surface with evenly distributed particles long the borehole wall). To specify a value for the average surface inclination $m$ is more difficult, since no values are known for powdery materials and moreover it may significantly depend on the fine structure and form of individual particles. For solid bodies in contact Antonetti et al. (1991) and Yovanovich et al. (1997) suggested a correlation formula for calculating $m$ as a 
function of $\sigma$, which has been experimentally verified for a surface roughness range of $0.216 \mu \mathrm{m} \leq \sigma<9.6 \mu \mathrm{m}$. For two conforming surfaces $(1,2)$ this relation is given as

$$
\begin{aligned}
& m_{1}=0.125 \sigma_{1}^{0.402} \\
& m_{2}=0.125 \sigma_{2}^{0.402}
\end{aligned}
$$

from which the average RMS inclination $m$ can be calculated by formula (7).

In order to illustrate the dependence of the thermal conductance between the sensor tube and the surrounding borehole wall we have evaluated formulae (3-9). Hereby we assume that the sensor's surface is coated by a dark material, so that its IR-emissivity is high and similar to that of the surrounding regolith $\left(\varepsilon_{1}=\varepsilon_{2} \approx 0.9\right)$, the roughness of the borehole surface is of the order of the average grain radius $\left(\sigma_{2}=50 \mu \mathrm{m}\right)$ and the roughness of the steel tube's outer surface is zero $\left(\sigma_{1}=0 \mu \mathrm{m}\right)$. Figure 9 shows the dependence of the total conductance $h$ from low pressures up to a value close to the assumed compaction strength of the lunar regolith $(220 \mathrm{kPa})$. The red line shows radiation conductance, which turns out to have a very low value in comparison to contact conductance, even though a worst case scenario was chosen $(T=394 \mathrm{~K}$, which was the maximum surface temperature measured at the Apollo 17 landing site) and high IR emissivities as given above. This choice results in radiation conductance values of about $15 \mathrm{Wm}^{-2} \mathrm{~K}^{-1}$. In reality temperatures along the borehole walls will be much lower (rather around $250 \mathrm{~K}$ ), with correspondingly smaller radiation conductances. It should be noted that the natural overburden pressure of the regolith in, say, $1 \mathrm{~m}$ depth lies rather at the lower end of the shown pressure scale. With the density value quoted in Table 1 and under lunar gravity one obtains an overburden
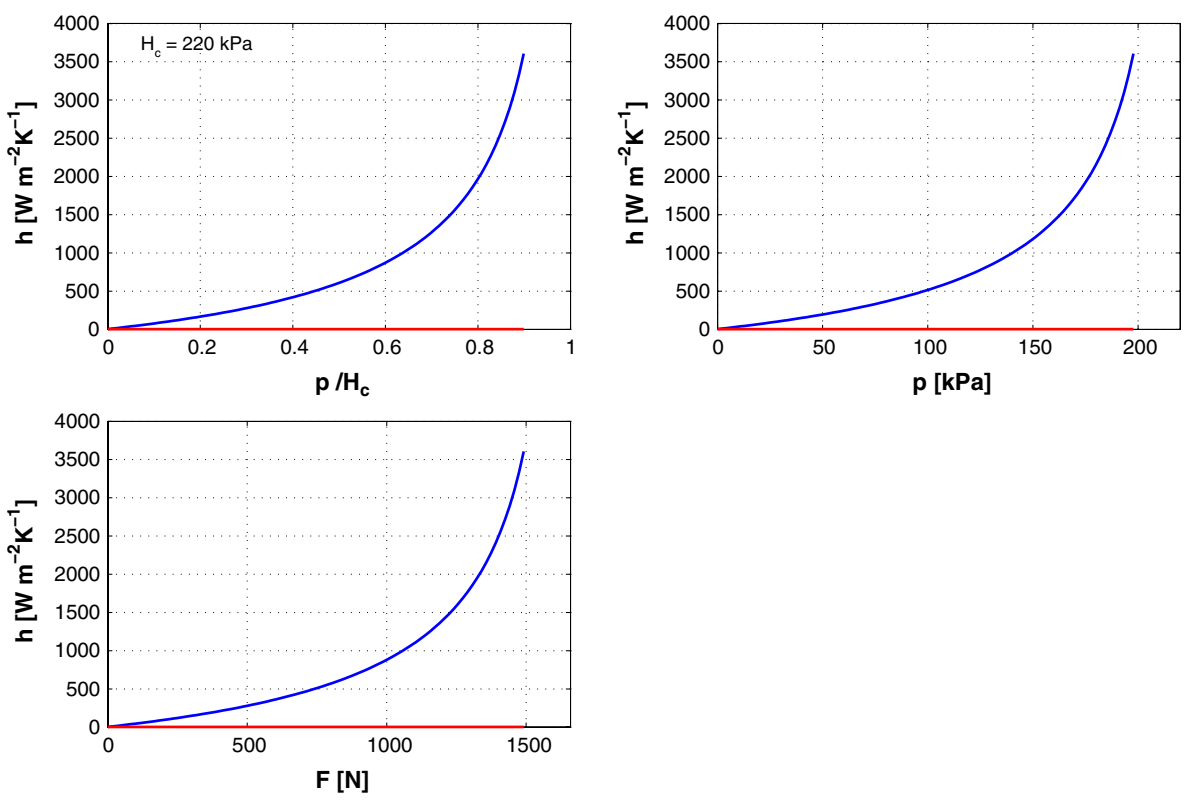

Fig. 9 Thermal conductance of the sensor-sample interface as a function of contact pressure. The blue line is the conductance due to the material contact between sensor and the soil, while the red line (not discernable from the $\mathrm{x}$-axis in these plots) is the contribution from mutual radiation for a temperature of $214 \mathrm{~K}$. Upper left panel: pressure in units of the compaction strength; upper right panel: pressure in $\mathrm{kPa}$; lower left panel: force acting over a a half lobe of the cylindrical outer mantle of a sensor with the dimensions shown in Fig. 7 
pressure in $1 \mathrm{~m}$ depth of $p \approx 2.8 \mathrm{kPa}$. This corresponds to a thermal conductance of $12.5 \mathrm{Wm}^{-2} \mathrm{~K}^{-1}$ and is comparable with the radiation contribution.

\section{Model Calculations}

As start-up for sensor development, we have set up numerical models for the two sensor scenarios described above, namely (a) a thick tube-like sensor attached to a borestem at the rear side of a drill bit, and (b) a thin sensor of the Hukseflux TP02 type at the front side of the drill bit (assuming that it can be pushed by motor force into the juvenile soil in front of the drillbit and retracted from the soil for drilling action). The following model calculations address primarily two points:

(1) How does the contact pressure of the sensor's outer surface against the borehole influence the temperature profile and-as a consequence-affect the measured thermal conductivity value of the surrounding material?

(2) Assuming a tube-shaped sensor, how does the presence of a borestem running through its interior and the mounting structure of the sensor influence the evolution of the temperature field in and around the sensor?

\subsection{Modelling Tube-Shaped Sensor}

In the first model geometry we consider a tube-shaped sensor with the dimensions of our prototype: outer diameter $16 \mathrm{~mm}$, inner diameter $10 \mathrm{~mm}$, length $300 \mathrm{~mm}$. The heater foil is sandwiched between two concentric steel tubes, each of which has a thickness of $1 \mathrm{~mm}$. Through the interior of the tube an $8 \mathrm{~mm}$ diameter borestem is running. Thus there is a $1 \mathrm{~mm}$ gap between borestem and sensor, across which heat transfer is only possible by radiation. The only material contact between sensor and borestem is via two thin teflon sleeves at the upper and lower end of the sensor, which connect the sensor to the drilling system. The whole system is placed vertically in a sample material (e.g. a lunar regolith analog material) which forms a concentric cylinder of $16 \mathrm{~cm}$ diameter around the sensor. For simplicity of the calculation, the parts of the borestem outside the sample cylinder are assumed to be thermally insulated. Figure 10 shows the geometry and a typical temperature distribution obtained by heating the sensor embedded in the sample with a constant power of $5 \mathrm{~W}$ for $20 \mathrm{~min}$. In Fig. 11 various profiles showing the response of the sensor and the surrounding medium to the heating are illustrated. The left hand panels show the radial temperature profiles at the end of the heating interval at a vertical position of $150 \mathrm{~mm}$ from the front end of the tube; the plots on the right hand panels show the heating curve of a point inside the sensor tube at a position $150 \mathrm{~mm}$ above its lower end as a function of time. Two model parameters are varied:

(i) The thermal conductivity of the surrounding sample material is changed from $0.02 \mathrm{Wm}^{-1} \mathrm{~K}^{-1}$ (regolith-like under vacuum), via $0.2 \mathrm{Wm}^{-1} \mathrm{~K}^{-1}$ (similar to dry sand under atmospheric conditions) to $2.0 \mathrm{Wm}^{-1} \mathrm{~K}^{-1}$ (bedrock-like).

(ii) In each of these panels the thermal conductance between sensor wall and regolith is varied from a low to a high value. The lowest value $\left(35 \mathrm{Wm}^{-2} \mathrm{~K}^{-1}\right)$ is of the order of the radiation conductance, while the highest value $\left(3500 \mathrm{Wm}^{-2} \mathrm{~K}^{-1}\right)$ is representative for a contact pressure in the range of the compression strength of the lunar regolith material as discussed before. In addition a thermal conductance value of $350 \mathrm{Wm}^{-2} \mathrm{~K}^{-1}$ is plotted, lying between these two extremes. Thus we think that the plots shown in Fig. 11 cover the interesting parameter space quite well. A sensor 


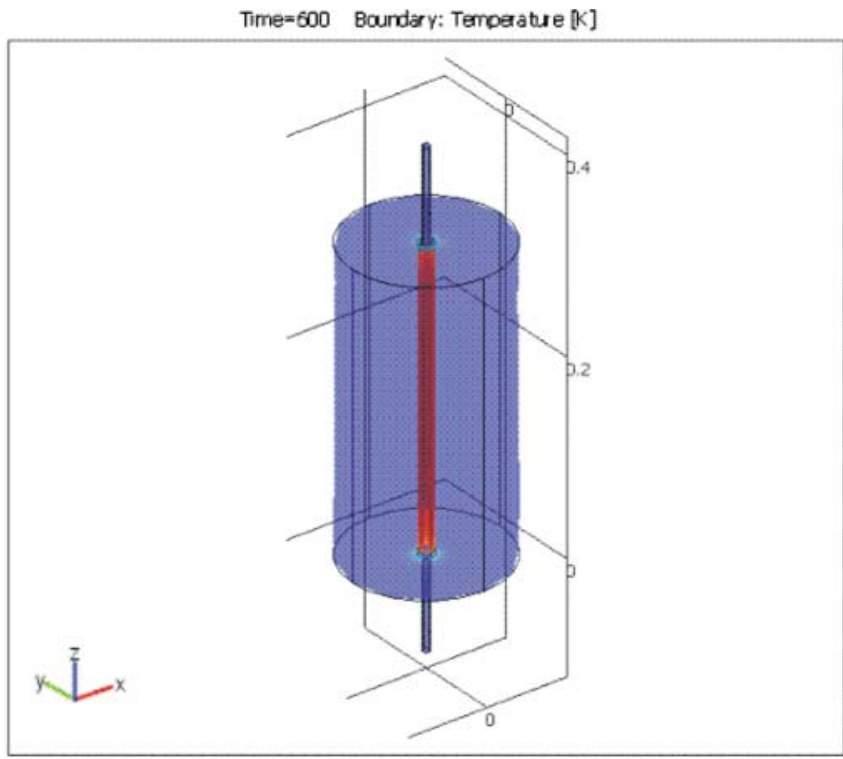

Max: 214.254

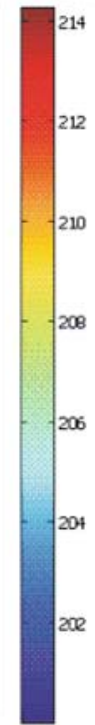

Time- 600 Boundary: Temperature [K]

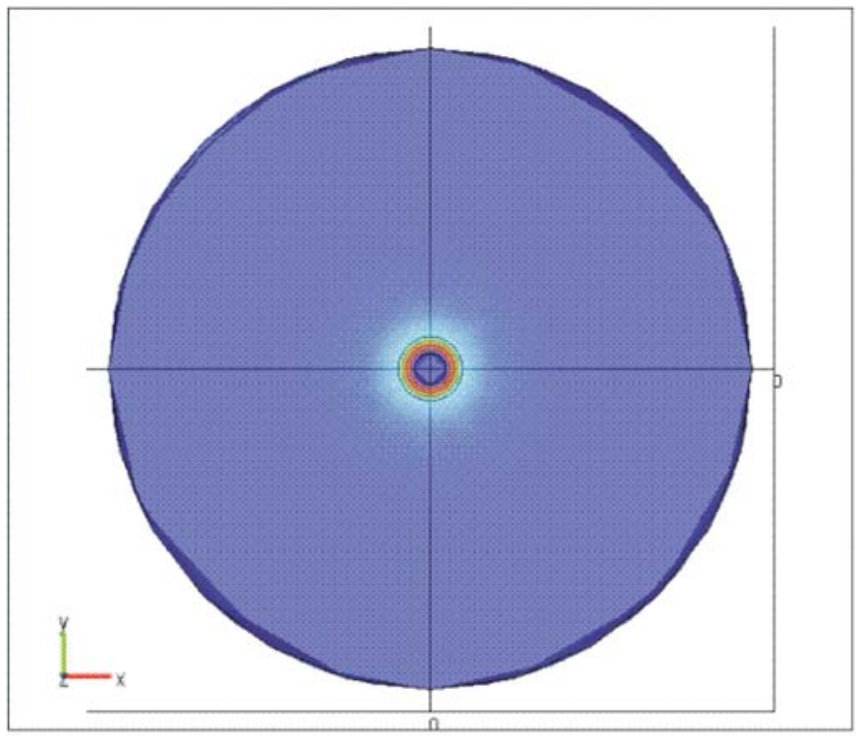

Min: 200

Max: 214.254

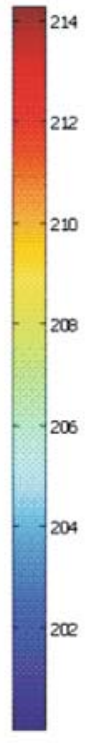

Mn: 200

Fig. 10 Model geometry for assessing the characteristics of the first suggested tube-like thermal conductivity sensor embedded in a regolith sample on the rear side of a drillbit: 3D-model including both the thermal contact resistance of the sensor to the regolith and the radiation interaction between the sensor and the bore stem fed through its central hole

positioned in a borehole inside the lunar regolith, which is mechanically pressed against the borehole walls with a pressure close to the regolith's compression strength is represented by the case $\lambda=0.02 \mathrm{Wm}^{-1} \mathrm{~K}^{-1}$ and $h=3500 \mathrm{Wm}^{-2} \mathrm{~K}^{-1}$. 

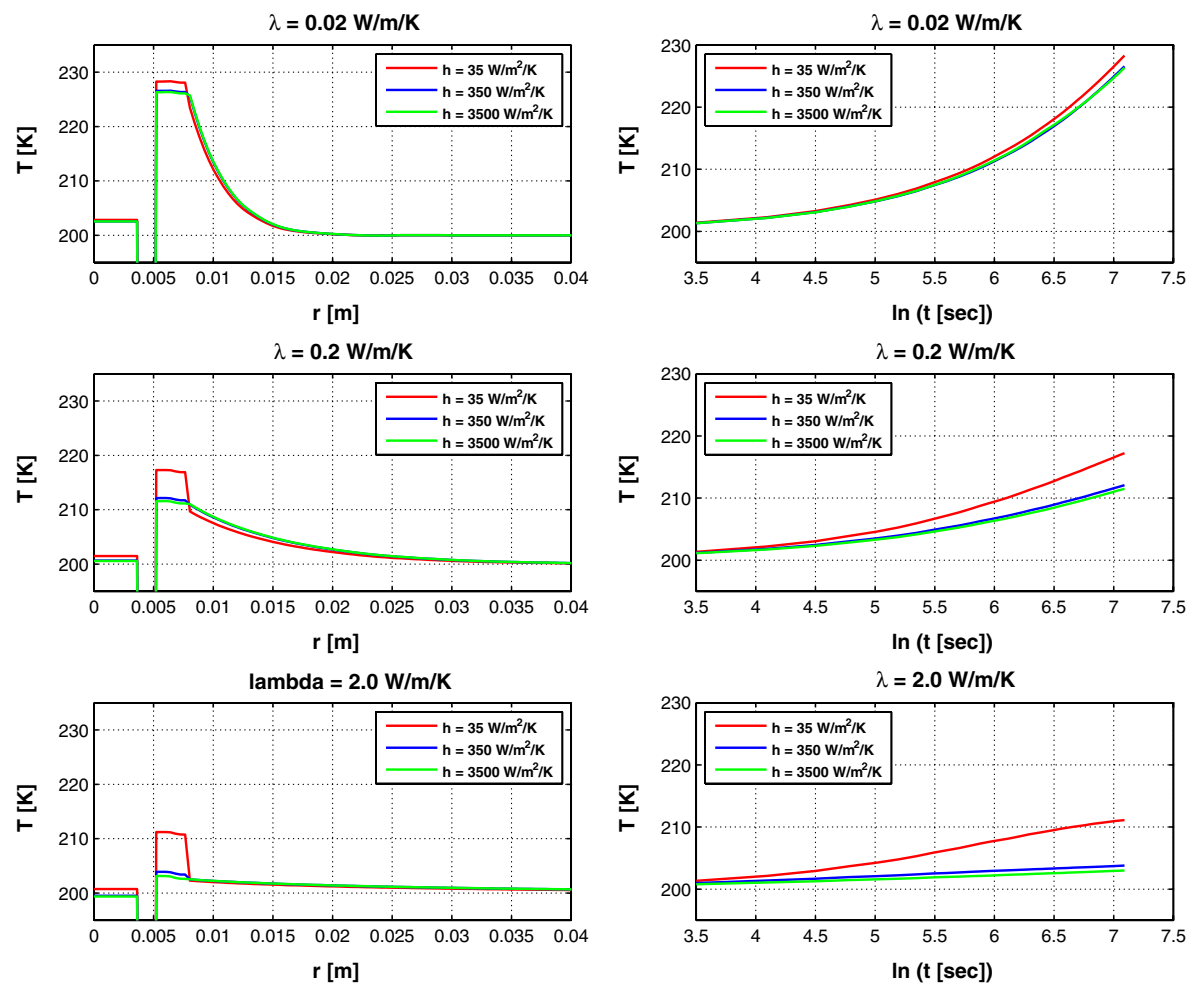

Fig. 11 Temperature distributions for the first scenario investigated for the implementation of a thermal sensor into a drillhead, as illustrated in Fig. 10, for different sample conductivities and thermal conductances between sensor and the surrounding sample material. The heating power was $5 \mathrm{~W}$ for a period of $20 \mathrm{~min}$

The second question posed above, namely the influence of a borestem running through the central hole of the thermal sensor, can also be answered by looking into the results of this model calculation. In the model the borestem is represented by an $8 \mathrm{~mm}$ diameter stainless steel rod. Figure 12 shows the temperature increase of the sensor tube in its central part for a configuration differing only in the presence or absence of the borestem. The sensor is inserted into a regolith analog sample with thermal conductivity $\lambda=0.02 \mathrm{Wm}^{-1} \mathrm{~K}^{-1}$. The results show that radiation interaction of the sensor with the borestem leads to a slightly slower increase of the heating curve, which needs to be taken into account in sensor calibration. However, the effect is not dramatic, although the calculation includes also the unavoidable material contact between sensor and borestem at the ends of the tube. According to Fig. 12 the deviation between the two cases after 20 min of heating with a power of $5 \mathrm{~W}$ is $<3 \mathrm{~K}$, which means $<10 \%$ in terms of the total temperature increase of the sensor during the heating period for any value of the thermal conductance between sensor and medium.

\subsection{Modelling Needle-Shaped Sensor Connected to Drillhead}

For the second scenario we assume that the thermal sensor consists of a thin steel needle of $2 \mathrm{~mm}$ diameter and $100 \mathrm{~mm}$ length, which is heated over the full length with a power of $0.2 \mathrm{~W}$ for $20 \mathrm{~min}$. It is assumed that it can be inserted into the juvenile soil at the bottom of 

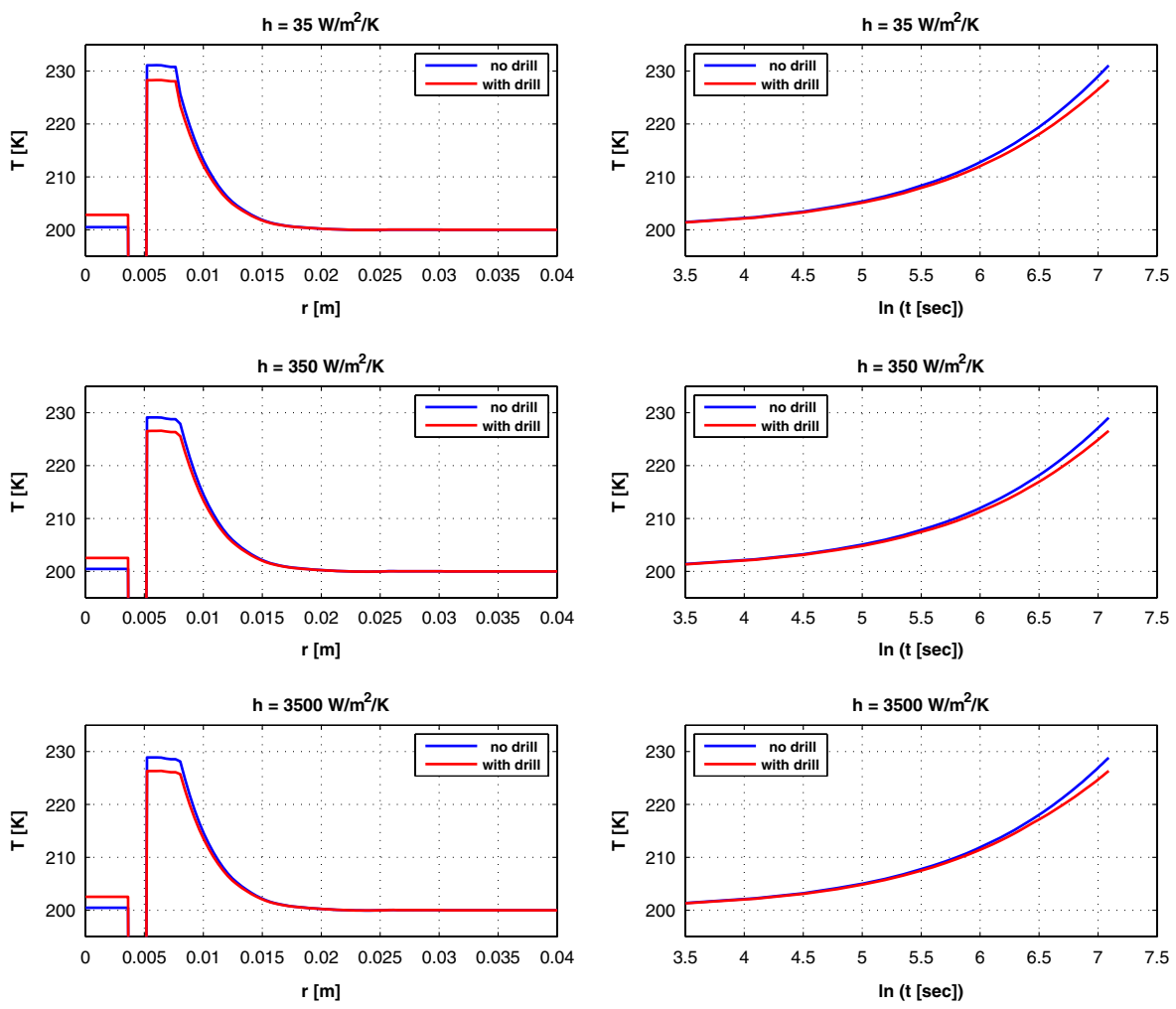

Fig. 12 Influence of the borestem running through the central hole of a tube-shaped thermal sensor on the temperature increase of the sensor in response to heating with a power of $5 \mathrm{~W}$ for a period of $20 \mathrm{~min}$

the drill hole by motor force. To mimic the thermal conditions we assume that both the sensor and the outer part of the drillhead have the thermal properties of stainless steel and that the thermal sensor can be moved up and down through a central hole tightly fitting the sensor diameter. In measuring position the sensor is in good contact with the undisturbed soil, while unavoidably some heat will also flow towards the drill head and the attached boring system. The assumed geometry is illustrated in Fig. 13. The sensor as well as the drillhead are embedded in a $16 \mathrm{~mm}$ diameter borehole, the sensor is in measuring position, i.e. extended from the bottom of the borehole into the underlying regolith. We assume that the borehead consists of a $16 \mathrm{~mm}$ outer diameter stainless steel shell, and is connected to the sensor needle via an embedded teflon sleeve. In upward direction the drillhead is connected with an $8 \mathrm{~mm}$ diameter borestem consisting of stainless steel. We simulate a worst case scenario by assuming that there is a high thermal conductance between the sensor and the drill head $\left(3500 \mathrm{Wm}^{-2} \mathrm{~K}^{-1}\right)$. The thermal conductance between the sensor and the sample material and the thermal conductivity of the sample material is varied in the same way as in the previous example. Figure 14 shows the results that can be directly compared to the corresponding curves for the tube-shaped sensor. Note, however, that because of the much smaller heat capacity of the needle sensor we have used a smaller heating power $(0.2 \mathrm{~W})$. With this choice the temperature increase of the sensor after $20 \mathrm{~min}$ of heating is still larger than for the tube-shaped sensor, but of the same order of magnitude. Again, for the lunar regolith case, the realistic parameter choice would be 


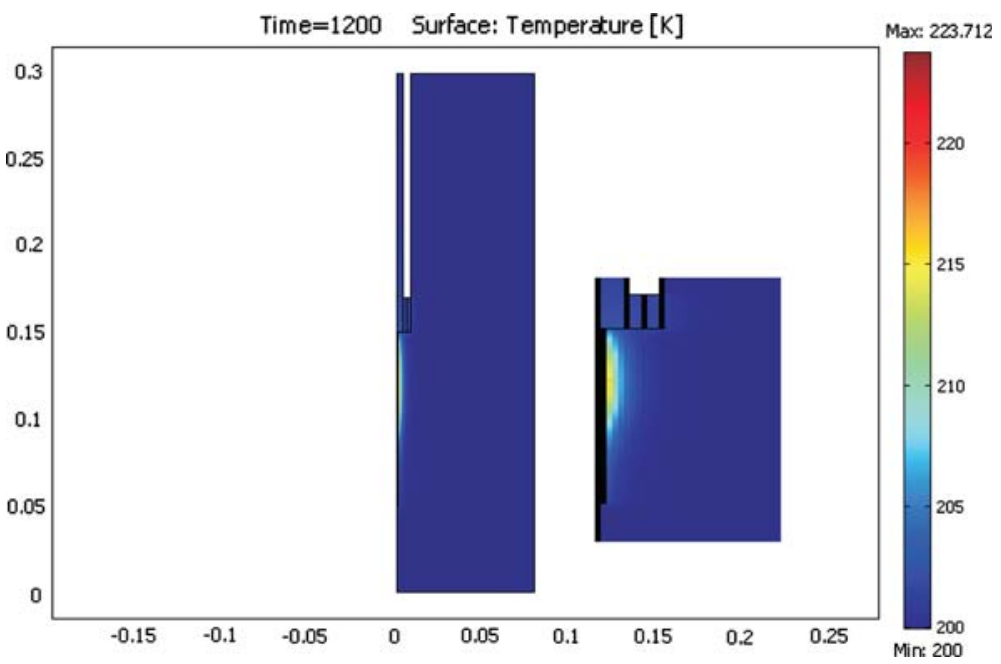

Fig. 13 Model geometry for assessing the characteristics of the second scenario suggested for the implementation of a thermal conductivity sensor into a drilling system: thin steel needle pushed into the soil ahead of the drillbit. The total simulation domain is shown in the correct scale in the central part of the figure, the inlet on the right side shows only the heated part around the needle. For better visibility of the temperature field the horizontal scale is exaggerated

$\lambda=0.02 \mathrm{Wm}^{-1} \mathrm{~K}^{-1}$ and $h=3500 \mathrm{Wm}^{-2} \mathrm{~K}^{-1}$. The advantage of this system lies in two facts: (i) There is no need to press the sensor against the soil other than by simply pushing it into the ground ahead of the drillhead, and (ii) evaluation of the thermal conductivity may be more straightforward, since the geometrical dimensions correspond more closely to the case of a line heat source. However, it certainly would be less robust and may fail by bending in case it approaches unexpectedly hard material during penetration.

\subsection{Evaluation Methods}

Finally we give a short discussion on the evaluation methods to be applied in order to derive reliable values for thermal parameters of the regolith from the heating curve of the sensor in response to a fixed power supply. The standard formula used to derive the thermal conductivity of a sample material from the measured temperature increase of a needle probe is

$$
\lambda=\frac{Q}{4 \pi\left(T_{2}-T_{1}\right)} \ln \left(\frac{t_{2}}{t_{1}}\right)
$$

where $Q$ is the heating power per unit length, $t_{1}$ and $t_{2}$ are two time points on the heating curve and $T_{1}$ and $T_{2}$ are the corresponding temperatures (Kömle et al. 2007, 2008a). However, this formula implies a couple of restrictions, since it is only strictly valid for a long and thin sensor where no heat flow in axial direction occurs. In this special case the thermal resistance between medium and sensor does not affect the derived value of $\lambda$. Using the simulation results for the needle-shaped sensor attached to a drillbit one can estimate the relative errors to be expected for the thermal conductivity measurement when using the simple evaluation formula (10). It turns out that for this configuration errors are small $(<1 \%)$ if the surrounding medium has a low thermal conductivity 

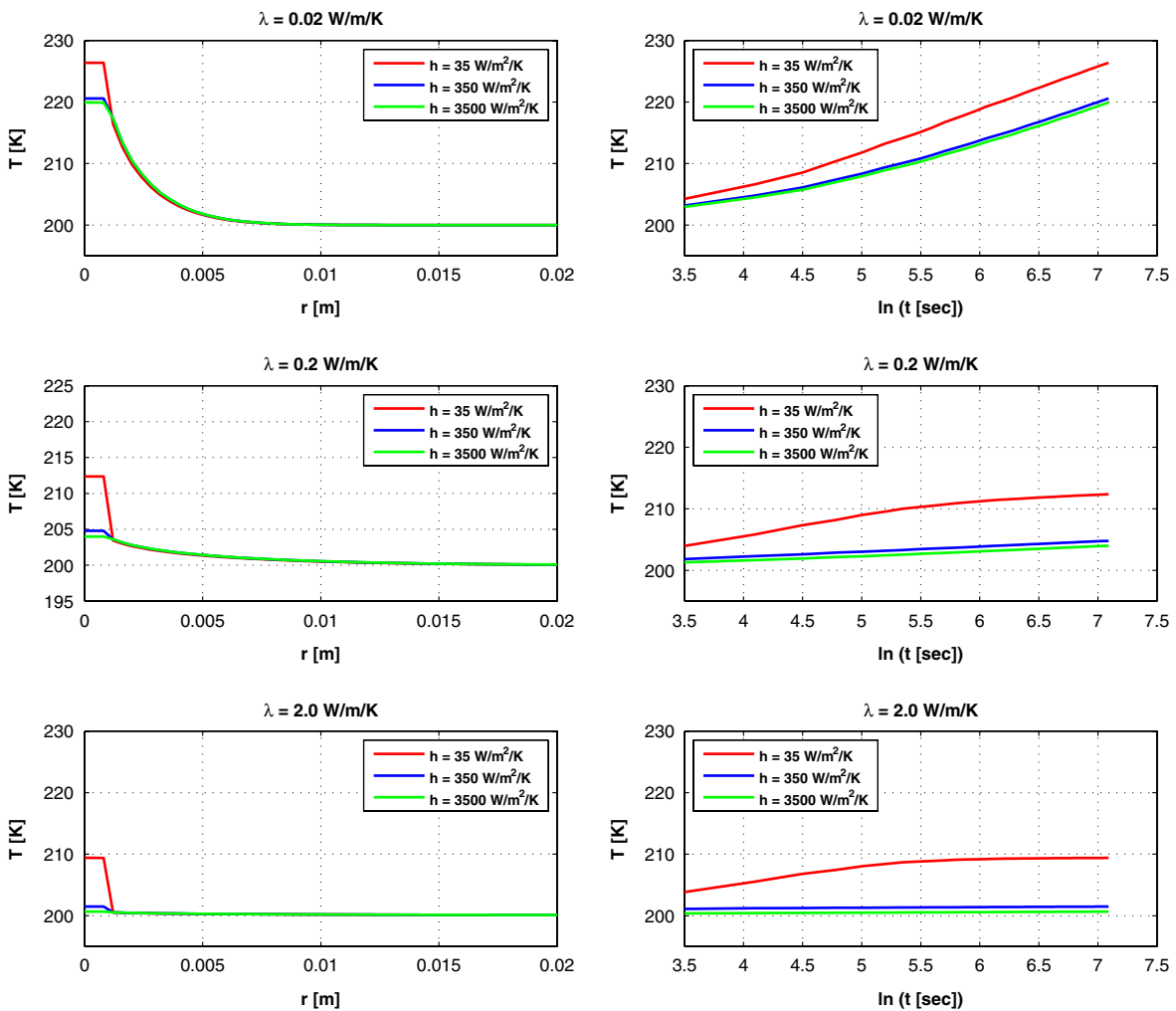

Fig. 14 Temperature distributions calculated for the second scenario investigated for the implementation of a thermal sensor into a drillhead, as illustrated in Fig. 13, for different sample conductivities and thermal conductances between sensor and the surrounding sample material The curves show the response of the needle-like sensor to heating with a power of $0.2 \mathrm{~W}$ for a period of $20 \mathrm{~min}$

( $\lambda=0.02 \mathrm{Wm}^{-1} \mathrm{~K}^{-1}$, the regolith case) and also there is only a weak dependence on the thermal conductance between sensor and medium. However, for a high thermal conductivity of the surrounding medium $\left(\lambda=2.0 \mathrm{Wm}^{-1} \mathrm{~K}^{-1}\right.$, the bedrock case $)$ the relative errors increase up to $36 \%$, if the contact between sensor and medium is poor ( $h=35 \mathrm{Wm}^{-2} \mathrm{~K}^{-1}$ ) but decrease to about $9 \%$ for a thermal conductance value of $h=3500 \mathrm{Wm}^{-2} \mathrm{~K}^{-1}$.

Evaluating thermal conductivities from measurements with the tube-shaped sensor demands a more sophisticated analysis, since its geometry deviates from the line heat source. This can also be seen from the fact that applying formula (10) in an analogous way leads to unacceptably large errors. However, the mathematical problem of predicting the temperature profile for a tube shaped sensor embedded in an infinitely extended medium is in principle analytically solved (see the standard textbook on Heat Conduction by Carslaw and Jaeger (1959)). Analytical formulae describing this case have been derived, for example, by Blackwell (1954) and by Kosky and McVey (1986). From these formulae the thermal conductivity of the medium and the thermal conductance at the interface can be determined simultaneously by applying a least squares fit to the measured temperature curve. The most general formula, as given by Kosky and McVey (1986) allows to calculate the temperature increase as a function of time over the whole heating period (early time as 
well as long time solution) by evaluating an integral depending on the thermal parameters $\lambda, \rho c$ and contact conductance $h$. Although the implementation of their algorithm is a bit cumbersome and demands some numerical effort, the tools to evaluate thermal conductivity values from measurements with tube-shaped sensors exist and can be used by exploiting the heritage from these early works.

\section{Summary and Outlook}

In this paper we have investigated two options how to integrate a drilling system with a thermal sensor and mount it as a payload on a lunar rover or as part of a stationary lunar geophysical package. Taking into account the severe weight limitations associated with such missions (for example ca. $90 \mathrm{~kg}$ for the Chang'e 2 lunar rover including all devices and payload), the driller (and probably also an attached sampling system) cannot easily be made as a traditional auger with several borestems mounted together. A variant of the described woodwasp drill may provide a low weight solution while still meeting the scientific requirements, which include a bore depth of about $2 \mathrm{~m}$.

The model calculations presented confirm that both options investigated for the implementation of a thermal sensor (thin needle in front of the drillhead or tube on the rear side) are feasible for determining the thermal properties of the subsurface regolith. In the latter case the sensor could be split into two separable half tubes that are pressed against the borehole walls during a measurement in order to ensure a low thermal resistance.

A problem which remains to be investigated is the thermal disturbance which is caused in the regolith by the drilling action itself. This depends of course on the type of drilling finally chosen. The relaxation time of the heat in the surrounding regolith may pose a problem especially on a rover mission, where the platform can stay on a fixed place only for a restricted time.

The next steps in this research will be the manufacture of the deployment device as described, the construction of one or several drill prototypes, and field testing of these devices together with the $\mathrm{BJ} 2$ rover prototype.

Acknowledgements This work was supported by the Austrian Fonds zur Förderung der wissenschaftlichen Forschung under project L317-N14. One of us (Hehua Ju) acknowledges funding support by the $\mathrm{Hi}^{-}$ Tech Research and Development Program of China (863 Program) in the frame of the following projects: Autonomous position and attitude determination technology based on IMU and Sun-compass for lunar rover (2006AA12Z307) and Lunar rover traction control technology (2005AA737080).

Open Access This article is distributed under the terms of the Creative Commons Attribution Noncommercial License which permits any noncommercial use, distribution, and reproduction in any medium, provided the original author(s) and source are credited.

\section{References}

C.C. Allen, J.C. Graf, D.S. McKay, Sintering bricks on the Moon. in Engineering, Construction, and Operation in Space IV. ed. by R.G. Galloway, S. Lokaj (American Society of Civil Engineers, New York, 1994), pp. 1220-1229

V.W. Antonetti, T.D. Whittle, R.E. Simons, An approximate thermal conductance correlation. in HTD-Vol. 170, Experimental/Numerical Heat Transfer in Combustion and Phase Change, pp. 35-42 (1991).

J.H. Blackwell, A transient-flow method for determination of thermal constants of insulating materials in bulk. J. Appl. Phys. 25, 137-144 (1954) 
W.D. Carrier III, G.R. Olhoeft, W. Mendell, Physical properties of the lunar surface. in LUNAR SOURCEBOOK-A User's Guide to the Moon. ed. by G.H. Heiken, D.T. Vaniman, B.M. French (Cambridge University Press, Cambridge, 1991), pp. 475-594

H.S. Carslaw, J.C. Jaeger, Conduction of Heat in Solids (Oxford University Press, Oxford, 1959)

H.-P. Ebert, V. Bock, O. Nilsson, J. Fricke, The hot wire method applied to porous materials of low thermal conductivity. High Temp-High Press 25, 391-402 (1993)

B.H. Foing, Concept studies for lunar landers and sample return missions: challenges for robotics. EPSC Abstracts, vol 2, EPSC2007-A-00422, European Planetary Science Congress, Potsdam, Germany, 2024 August 2007.

Y. Gao, A. Ellery, J. Vincent, Bio-inspired drill for planetary sampling-literature survey, conceptual design and feasibility study. AIAA J. Spacecraft Rockets 44(3) (2007).

M. Grujicic, C.L. Zhao, E.C. Dusel, The effect of thermal contact resistance on heat management in the electronic packaging. Appl. Surf. Sci. 246, 290-302 (2005)

R. Haenel, L. Rybach, L. Stegena, Handbook of Terrestrial Heat-Flow Density Determination. Solid Earth Sciences Library (Kluwer Academic Publishers, Dordrecht, 1988)

G.H. Heiken, D.T. Vaniman, B.M. French (ed.): LUNAR SOURCEBOOK-A User's Guide to the Moon. (Cambridge University Press, Cambridge, 1991)

B. Hufenbach, European long-term strategy for space exploration and the role of the Moon/robotic lunar missions. European Workshop on Lunar Landers, ESTEC, Noordwijk, The Netherlands, 15-16 December 2005

H.H. Ju, P.J. Cui, H.J. Liu, Autonomous behavior agent based lunar rover motion planning and control. Acta Automat. Sin. 32, 704-716 (2006)

N.I. Kömle, B. Hui, W.J. Feng, R. Wawrzaszek, E. Hütter, P. He, W. Marczewski, B. Dabrowski, K. Schröer, T. Spohn, Thermal conductivity measurements of road construction materials in frozen and unfrozen state. Acta Geotech. 2, 127-138 (2007). doi:10.1007/s11440-007-0032-1

N.I. Kömle, E. Kaufmann, G. Kargl, Y. Gao, R. Xu, Development of thermal sensors and drilling systems for lunar and planetary regoliths. Adv. Space Res. 42, 363-368 (2008a). doi:10.1016/j.asr. 2007.02.088

N.I. Kömle, P. Weiss, K.L. Yung, Considerations on a suction drill for lunar surface drilling and sampling: I. Feasibility study. Acta Geotech. 3, 201-214 (2008b). doi:10.1007/s11440-008-0076-X

J.A. Koski, D.F. McVey, Application of parameter estimation techniques to thermal conductivity probe data reduction. in Thermal Conductivity, vol 17, ed. by J.G. Hust (Plenum Press, New York 1986), pp. 587-600

A.J.R. Langevin, The evolution of the lunar regolith. Annu. Rev. Earth Planet. Sci. 5, 449-489 (1977). doi: 10.1146/annurev.ea.05.050177.002313

M.G. Langseth, S.P. Clark, J. Chute, S.J. Keihm, A.E. Wechsler, The Apollo 15 lunar heat-flow experiment. Moon 4, 390-410 (1972)

M.G. Langseth, S.J. Keihm, K. Peters, Revised lunar heat-flow values. in Proceedings of the 7th Lunar Science Conference, pp. 3143-3171 (1976)

K. Matsumoto, N. Kamimoria, Y. Takizawa, M. Kato, M. Oda, S. Wakabayashi, S. Kawamoto, T. Okada, T. Iwata, M. Ohtake, Japanese lunar exploration long-term plan. Acta Astronaut. 59, 68-76 (2006)

D.S. McKay, G. Heiken, A. Basu, G. Blanford, S. Simon, R. Reedy, B.M. French, J. Papike, The lunar regolith. in LUNAR SOURCEBOOK-A User's Guide to the Moon. ed. by G.H. Heiken, D.T. Vaniman, B.M. French (Cambridge University Press, Cambridge, 1991), pp. 475-594

National Research Council of the National Academies (US): The Scientific Context for the Exploration of the Moon (The National Academies Press, Washington, DC, 2006) http://www.nap.edu

C.R. Neal: Development of a Lunar Geophysical Instrument Package. ROSES 2006 Proposal to NASA, September 2006

NOAA Satellite and Information Service: Borehole Temperatures and Climate Reconstruction Database. http://www.ncdc.noaa.gov/paleo/borehole/core.html (last update 2005)

C.C. Pilbeam, J.R. Vaisnys, Contact thermal conductivity in lunar aggregates. J. Geophys. Res. 78, 52335236 (1973)

R.A. Schultz, R. Siddhartan, Strength of lunar soil using the cam cap approach. Abstract \#1127 published in Lunar and Planetary Science XXXVIII, CD-ROM (http://www.lpi.usra.edu/meetings) (Lunar and Planetary Institute, Houston, 2007)

T. Spohn, K. Seiferlin, A. Hagermann, J. Knollenberg, A.J. Ball, M. Banaszkiewicz, J. Benkhoff, S. Gadomski, J. Grygorczuk, M. Hlond, G. Kargl, E. Kührt, N. Kömle, W. Marczewski, J.C. Zarnecki (2007) MUPUS - A thermal and mechanical properties probe for the Rosetta Lander PHILAE. Space Sci. Rev. 128, 339-362

D. Vaniman, R. Reedy, G. Heiken, G. Olhoeft, W. Mendell, The lunar environment. in LUNAR SOURCEBOOK-A User's Guide to the Moon, ed. by G.H. Heiken, D.T. Vaniman, B.M. French (Cambridge University Press, Cambridge, 1991), pp. 475-594 
J.F.V. Vincent, M.J. King, The mechanism of drilling by wood wasp ovipositors. Biomimetics 3(4), 187201 (1995)

A.E. Wechsler, P.E. Glaser, Pressure effects on postulated lunar materials. Icarus 4, 335-352 (1965)

M.M. Yovanovich, Conduction and thermal contact resistances (conductances). in Heat Transfer, ed. by W.M. Rohsenov, J.P.Hartnett, Y.I. Cho, pp. 3.1-3.73 (1998)

M.M. Yovanovich, J.R. Culham, P. Teestra, Calculating interface resistance (1997), http://electronicscooling.com/articles/1997/may/article3.php. Retrieved in Aug 2007

K. Zacny, D. Glaser, P. Bartlett, K. Davies, J. Wilson, Drilling results in ice-bound simulated lunar regolith (FJS-1) as part of the construction and utilization explorer project (CRUX). Abstract \#2226 published in Lunar and Planetary Science XXXVII, CD-ROM (http://www.lpi.usra.edu/meetings) (Lunar and Planetary Institute, Houston, 2006).

K. Zacny, G. Mungas, L. Parrington, C. Mungas, D. Fisher, Pneumatic drill and excavator for planetary exploration. Contribution 3010 Presented at the Seventh International Conference on Mars, 9-13 July 2007, Pasadena, California (2007) 